\title{
Prevalence of Carbapenem Resistance and their Genotypic Profile among Gram-Negative Bacteria in a Tertiary Care Hospital in Western India
}

\author{
Parijat Das $^{1 *}$, Kumar Anand Shrutiraaj ${ }^{2}$, Manish Ranjan ${ }^{1}$ and Sourav Sen ${ }^{1}$ \\ 'Department of Microbiology, Armed Forces Medical College, Pune, 411040, India. \\ ${ }^{2}$ Department of Medicine, Armed Forces Medical College, Pune, 411040, India.
}

\begin{abstract}
Background: Resistance to carbapenems due to carbapenemases has been increasingly noticed worldwide. Detection of carbapenemases among Gram-negative bacteria (GNB) is important for both clinicians and infection control practitioners. Both phenotypic and molecular methods can be used for detection of Carbapenemases production. Molecular methods although the gold standard for detection of carbapenemases are not used routinely as they might not be immediately available coupled with expertise required, cost and infrastructure incurred and limited by the number of targets detected.

Methods: Consecutive non-repeat gram negative isolates isolated from various clinical specimens from intensive care unit (ICU) were included in the study. Antimicrobial susceptibility testing was done on Mueller Hinton's agar by Kirby Bauer Disc diffusion method as per the Clinical and Laboratory Standards Institute (CLSI) guidelines. Isolates resistant to Meropenem were further screened for carbapenemase producing genes using multiplex polymerase chain reaction (PCR). The results were statistically analysed.

Result: A total of 350 gram negative bacteria were screened for carbapenem resistance. Carbapenem resistance was found in 109 GNB. The metallo- $\beta$-lactamases were most common carbapenemases followed by KPC.

Conclusion: Carbapenemase producing bacteria are a major threat of the 21 st century. Preventing emergence and spread of these pathogens through strict infection control practices, judicious use of antibiotics and early and timely detection will contribute in preserving carbapenems, the last resort antibiotics.
\end{abstract}

Keywords: Carbapenem-Resistant Organisms, Metallo- $\beta$-Lactamase, Enterobacteriaceae, Polymerase Chain Reaction

\section{Introduction}

A major public health issue worldwide has been the emergence of resistance to carbapenems due to production of carbapenem hydrolysing beta-lactamases (carbapenemases) which renders them resistant to all $\beta$-lactam agents including penicillins, cephalosporins, monobactams, and carbapenems ${ }^{[1]}$. The genes encoding for carbapenemases usually belong to Ambler class B metallo- $\beta$-lactamases (MBLs) or to Ambler class A KPCtype enzymes and occasionally Ambler class D.

The carbapenemases characterized from gram negative bacteria include KPC, SME, IMI, VIM, NDM, OXA type enzymes and GES. The majority of carbapenem resistance among gram negative bacteria is attributed to MBL and KPC expression. The KPC- $\beta$ lactamase occurs most commonly in K. pneumoniae, but it has also been reported in other species of Enterobacteriaceae (Klebsiella oxytoca, Enterobacter spp., Escherichia coli, Salmonella spp., Citrobacter freundii, and Serratia spp.) and Pseudomonas aeruginosa ${ }^{(14)}$.

The reason for our interest in such genes encoding for carbapenemases is because of the fact that they are present on conjugative plasmids thereby increasing their potential for rapid dissemination and causing life threatening infections by multi drug resistant bacteria for which we might not have a cure. Given the dwindling therapeutic options capable of combatting carbapenemase producing microorganisms, knowledge of carbapenemases producing microorganisms will be the crucial step in controlling their spread and ensuring a favorable clinical outcome ${ }^{[2]}$.

The situation is further worsened by the fact that such microorganisms are frequently resistant to other groups of antibiotics ${ }^{[3]}$. The fact that even automated systems are not $100 \%$ successful in detection of carbapenemase-mediated resistance is worrisome ${ }^{[4]}$. Although molecular methods are highly efficient, they require costly infrastructure and incur high running cost which makes them impracticable for routine testing.

\section{Aim and Objectives}

Aim: To detect Carbapenemases production in Gram negative bacteria from clinical samples of patients admitted in ICU and genotypic profiling of carbapenemases in a Tertiary Care Centre. Objectives: To determine 
the prevalence of Gram negative bacteria resistant to carbapenems in samples from ICU patients. Genotypic profiling of the carbapenem resistant isolates.

\section{Materials and Methods}

This prospective study was carried out in Tertiary care hospital in Western India. Clearance from the Institutional Ethical Committee was obtained prior to commencement of the study. Informed written consent was taken from all participant patients of the study. Over a period of eighteen months from Jan 2016 - Jun 2017, 350 consecutive nonrepeat gram negative isolates isolated from various clinical specimens from intensive care unit (ICU) were included in the study.

All specimens except urine were inoculated on MacConkey agar and Blood agar. Urine samples were inoculated on Cystine Lactose Electrolyte Deficient agar (CLED). The inoculated plates were incubated aerobically at $37^{\circ} \mathrm{C}$ for 18 hours. The blood culture specimens were inoculated in blood culture bottles and incubated aerobically at $37^{\circ} \mathrm{C}$ in automated blood culture systems. On receipt of a positive signal, the particular specimen was sub cultured from the blood culture bottle onto blood and MacConkey agar and incubated as above.

On completion of the required incubation time, bacterial identification wascarried outbaseduponcolonymorphology, Gram stain, lactose fermentation, production of acid/ gas and a battery of other biochemical tests such as catalase and oxidase test, nitrate reduction, sugar fermentation, triple sugar iron (TSI) test, triphenyl tetrazolium chloride motility (TTC) medium, decarboxylase test etc.

The antimicrobial susceptibility testing was done by the Kirby Bauer disc diffusion method on Muller Hinton agar and commercially available antimicrobial discs (HiMedia) were used. The sizes of the zones of inhibition were interpreted as per CLSI: Performance Standards for Antimicrobial Susceptibility Testing: Twenty Sixth Informational Supplement ${ }^{[13]}$. All gram negative isolates resistant to Meropenem were further tested for production of carbapenemases.

\section{Molecular methods}

All Meropenem resistant isolates and 50 randomly chosen isolates which were sensitive to carbapenems by disc diffusion method were subjected to DNA extraction with HiPurA ${ }^{\mathrm{TM}}$ Plasmid DNA Miniprep Purification Kit (HIMEDIA) followed by conventional PCR technique for detection of resistance genes $b l a_{\mathrm{NDM}-1}, b l a_{\mathrm{VIM}}, b l a_{\mathrm{IMP}}, b l a_{O X A}$ and $b l a_{\mathrm{KPC}}$ (Table-1) which were purchased from Eurofins Genomic Pvt Ltd.

\section{Statistical Analysis}

All the data was entered on Excel sheet and analyzed by using SPSS version 22 software.

\section{Result}

A total of 350 isolates of Gram negative bacteria from various clinical samples were collected. All the clinical isolates were tested for resistance to Carbapenem class of drugs by Kirby-Bauer disc diffusion method.

Distribution gender wise: Out of the 350 samples 212 $(62 \%)$ were from male patients and $138(38 \%)$ were from female patients as shown below in Figure-1.

Species wise distribution: Out of 350 samples the majority were Enterobacteriaceae (E.coli and Klebsiella spp and Citrobacter spp) Pseudomonas spp and Acinetobacter spp were also isolated. Species wise distribution of isolates is shown below in Figure-2.

Sample wise distribution: The majority of samples were Urine followed by, Pus, Respiratory samples and Blood respectively. Distribution of samples is shown below in Figure-3.

Age distribution: Out of the 350 samples $37 \%$ of the samples were from $16 \mathrm{Y}-40 \mathrm{Y}$ followed by $35 \%$ belonging to $41 \mathrm{Y}-65 \mathrm{Y}$. Age wise distribution of samples is shown below in Figure-4.

Meropenem Sensitivity pattern: Out of 350 isolates 231 were sensitive, 10 were intermediate and 109 isolates were resistant to Meropenem by the Kirby bauer disc diffusion method.

Species wise distribution of Meropenem resistance: Species wise distribution of resistant isolates is shown in Table-2.

PCR: All the 109 resistant samples were subjected to PCR for the detection of carbapenemase genes. In addition randomly selected 50 isolates which were sensitive to carbapenems by disc diffusion were subjected to PCR.

Genotypic Profiling of Carbapenemases: The results of PCR are shown in Table-3. Gel electrophoresis is shown in Figure-5 and Figure-6.

The total of last row is more than $100 \%$ as few (04) isolates had two carbapenemase genes

\section{Discussion}

In our study, a total of 350 isolates of Gram negative bacteria from various clinical samples collected during a period of eighteen months from Jan 2016 to June 2017 were tested for resistance to carbapenem class of drugs. In this study, out of 350 isolates, 166 (47.4\%) were E.coli 
Table 1: PCR targets for carbapenemases.

\begin{tabular}{|c|c|c|}
\hline Primer name & Primer sequence & PCR product size \\
\hline IMP2-F & GGAATAGAGTGGCTTAAYTCTC & \multirow[t]{2}{*}{ 232bp } \\
\hline IMP2R-2 & CCAAACYACTASGTTATCT & \\
\hline VIM-F & GATGGTGTTTGGTCGCATA & \multirow[t]{2}{*}{$390 \mathrm{bp}$} \\
\hline VIM-R & CGAATGCGCAGCACCAG & \\
\hline OXA-48A & TATATTGCATTAAGCAAGGG & \multirow[t]{2}{*}{$800 \mathrm{bp}$} \\
\hline OXA-48B & CACACAAATACGCGCTAACC & \\
\hline NDMf & CACCTCATGTTTGAATTCGCC & \multirow[t]{2}{*}{$984 b p$} \\
\hline NDMr & CTCTGTCACATCGAAATCGC & \\
\hline KPCyF & TGTCACTGATCGCCGTC & \multirow[t]{2}{*}{$1011 b p$} \\
\hline KPCyR & CTCAGTGCTCTACAGAAAACC & \\
\hline
\end{tabular}

Table 2: Species-wise distribution of Meropenem Resistant isolates.

\begin{tabular}{|l|l|l|l|}
\hline Organism & Total & Resistant Isolates & Percentage Resistance \\
\hline Acinetobacter baumanii & 30 & 21 & $70 \%$ \\
\hline Burkholderia spp & 3 & 0 & - \\
\hline Citrobacter freundii & 15 & 6 & $40 \%$ \\
\hline Citrobacter koseri & 1 & 0 & - \\
\hline Escherichia coli & 166 & 33 & $20 \%$ \\
\hline Enterobacter spp & 7 & 2 & $28.6 \%$ \\
\hline Klebsiella oxytoca & 2 & 0 & - \\
\hline Klebsiella pneumoniae & 53 & 22 & $41.5 \%$ \\
\hline Pseudomonas spp & 53 & 23 & $43.4 \%$ \\
\hline Proteus vulgaris & 7 & 1 & $14.3 \%$ \\
\hline Proteus mirabilis & 9 & 0 & - \\
\hline Misc & 4 & 1 & - \\
\hline TOTAL & $\mathbf{3 5 0}$ & $\mathbf{1 0 9}$ & $\mathbf{3 1 . 1 4 \%}$ \\
\hline
\end{tabular}

Table 3: Genotypic profile of Carbapenem resistant isolates.

\begin{tabular}{|c|c|c|c|c|c|}
\hline \multirow{2}{*}{$\begin{array}{l}\text { Meropenem resistant } \\
\text { organisms }\end{array}$} & \multicolumn{5}{|c|}{ Carbapenemase - encoding genes } \\
\hline & NDM-1 (\%) & KPC (\%) & OXA 48 type (\%) & IMP (\%) & VIM (\%) \\
\hline Escherichia coli $(\mathrm{n}=33)$ & 12 & 24 & - & - & 1 \\
\hline Klebsiella spp $(\mathrm{n}=22)$ & 06 & 16 & 2 & - & - \\
\hline Pseudomonas spp $(n=23)$ & 19 & - & 4 & - & - \\
\hline Acinetobacter spp $(n=21)$ & 12 & - & 9 & - & - \\
\hline Citrobacter spp $(n=6)$ & 3 & - & 2 & - & 1 \\
\hline Enterobacter spp $(\mathrm{n}=2)$ & - & - & 1 & - & 1 \\
\hline Misc spp. $(n=2)$ & 2 & - & - & - & - \\
\hline Total = $113(109)$ & $54(49.5 \%)$ & $38(34.8 \%)$ & $18(16.5 \%)$ & - & $3(2.7 \%)$ \\
\hline
\end{tabular}




\section{$n=350$}

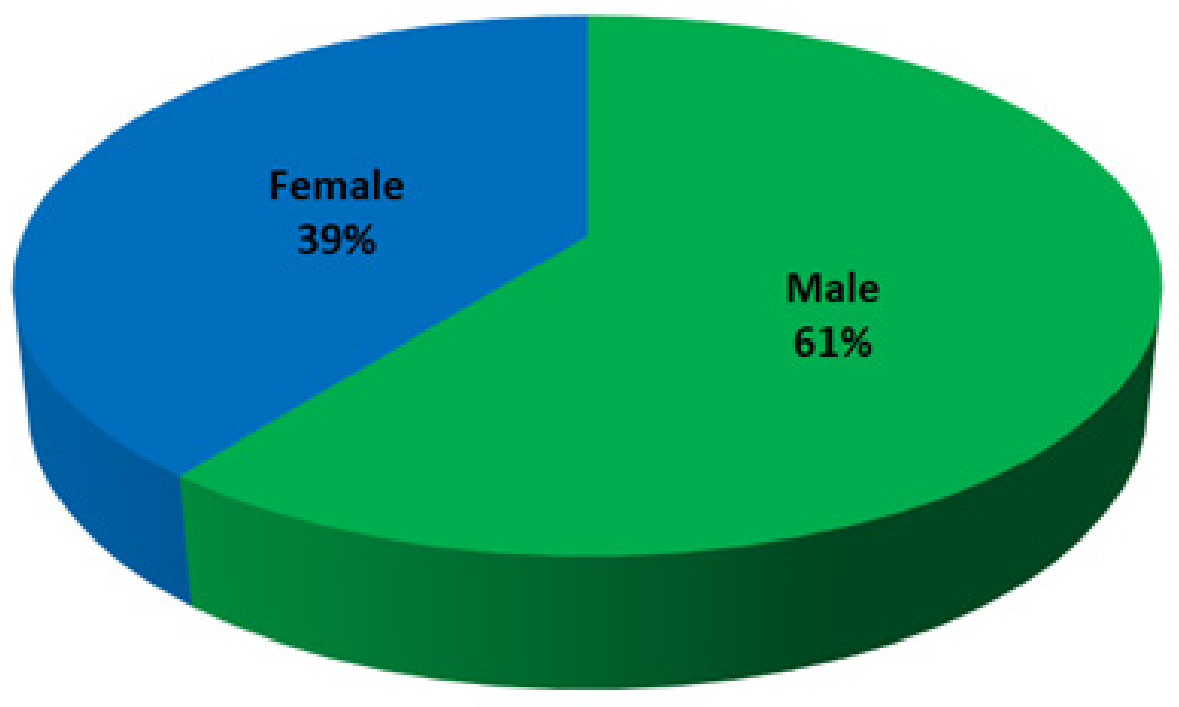

Fig. 1: Gender-wise distribution of isolates.

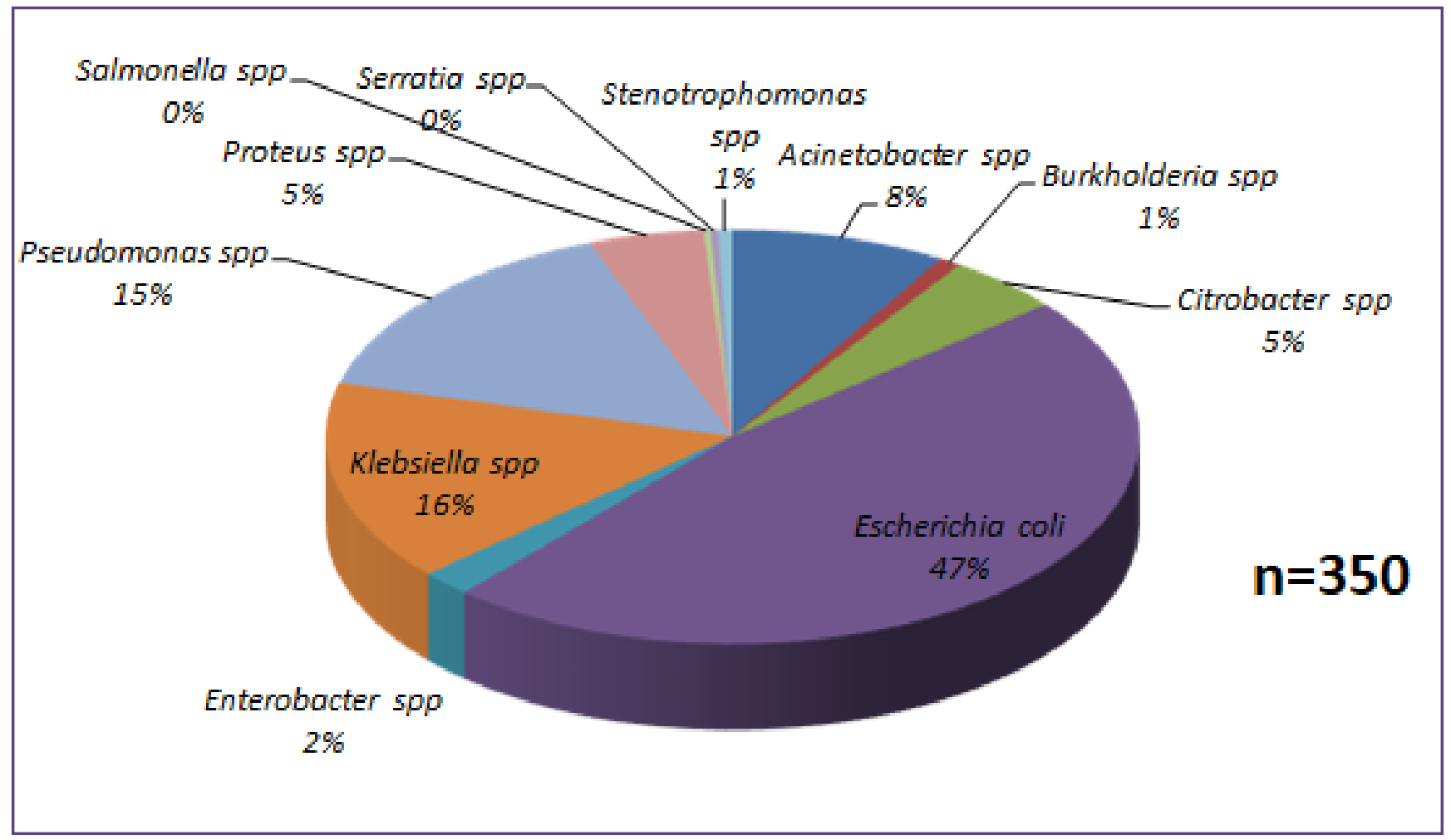

Fig. 2: Species-wise distribution of isolates. 


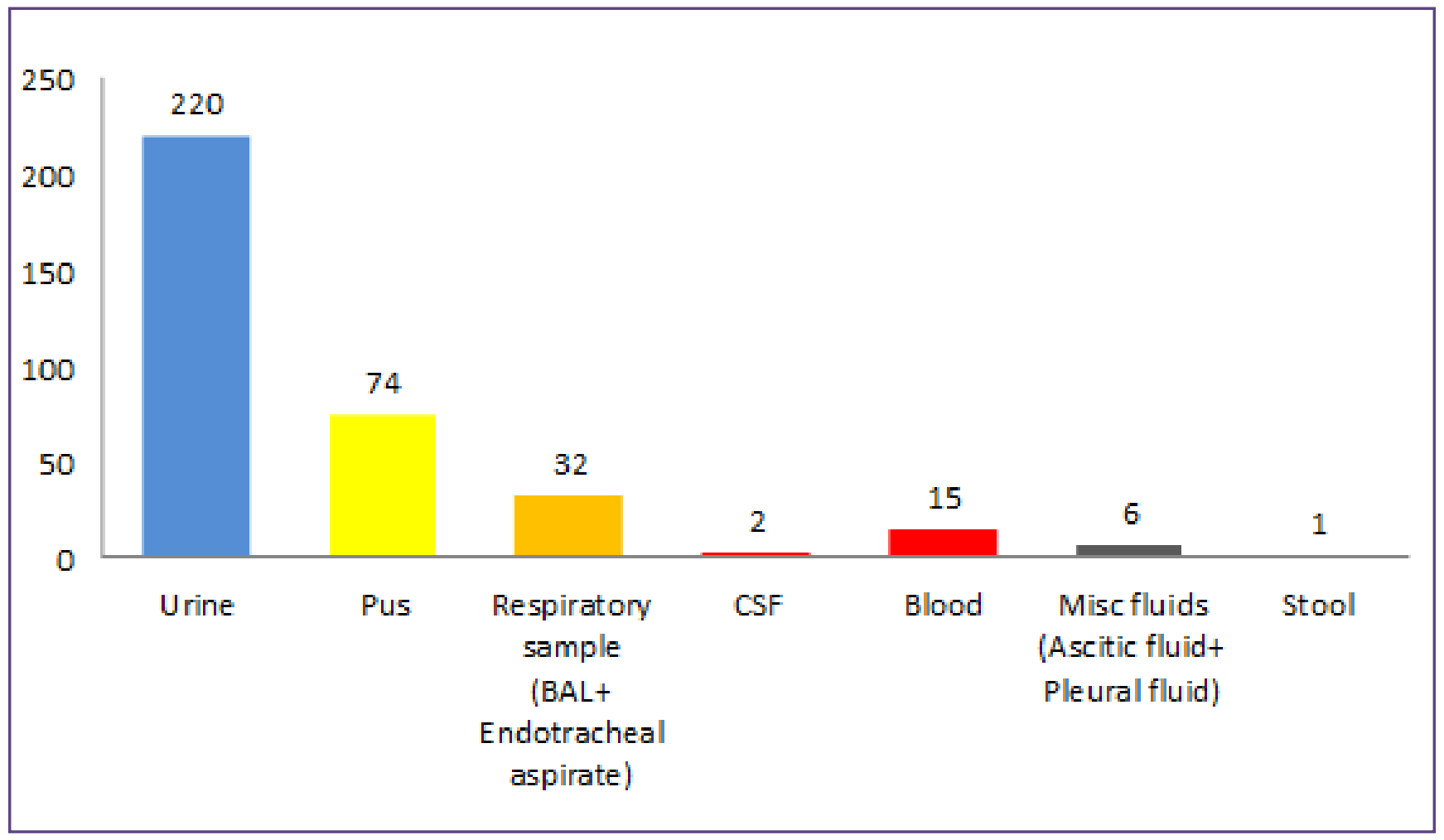

Fig. 3: Sample-wise distribution of isolates.

\section{Age}

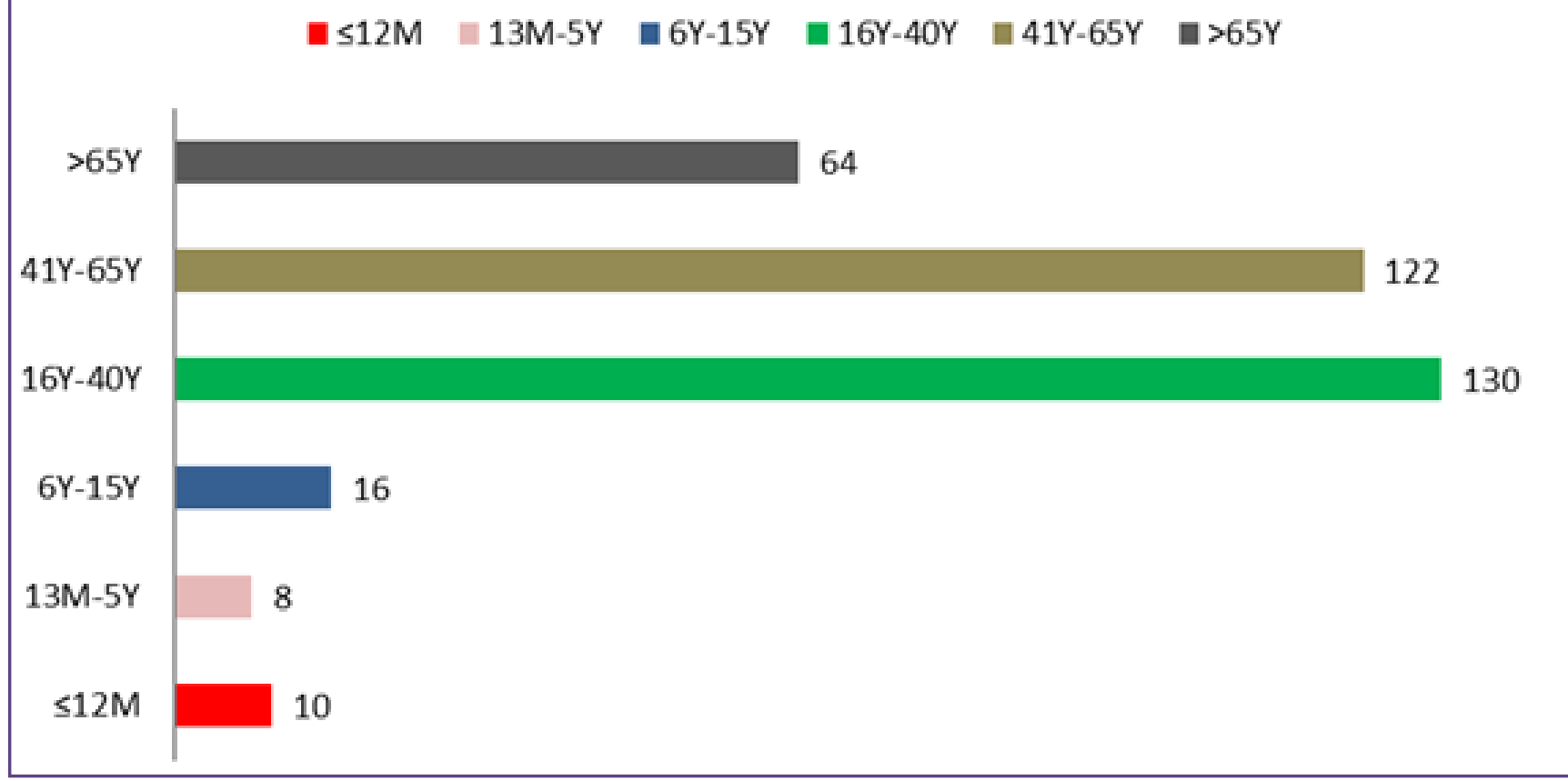

Fig. 4: Age-wise distribution of Samples. 


\section{GEL ELECTROPHORESIS}

$\begin{array}{llllllllllll}N C & 1 & 2 & 3 & 4 & 5 & 6 & 7 & \text { PC } & 8 & \text { MW }\end{array}$

\section{0 bp}

\section{$1011 \mathrm{bp}$}

\section{$390 \mathrm{bp}$}

Fig. 5: Gel Electrophoresis I PC-Positive control NC- Negative control MW-molecular weight ladder.

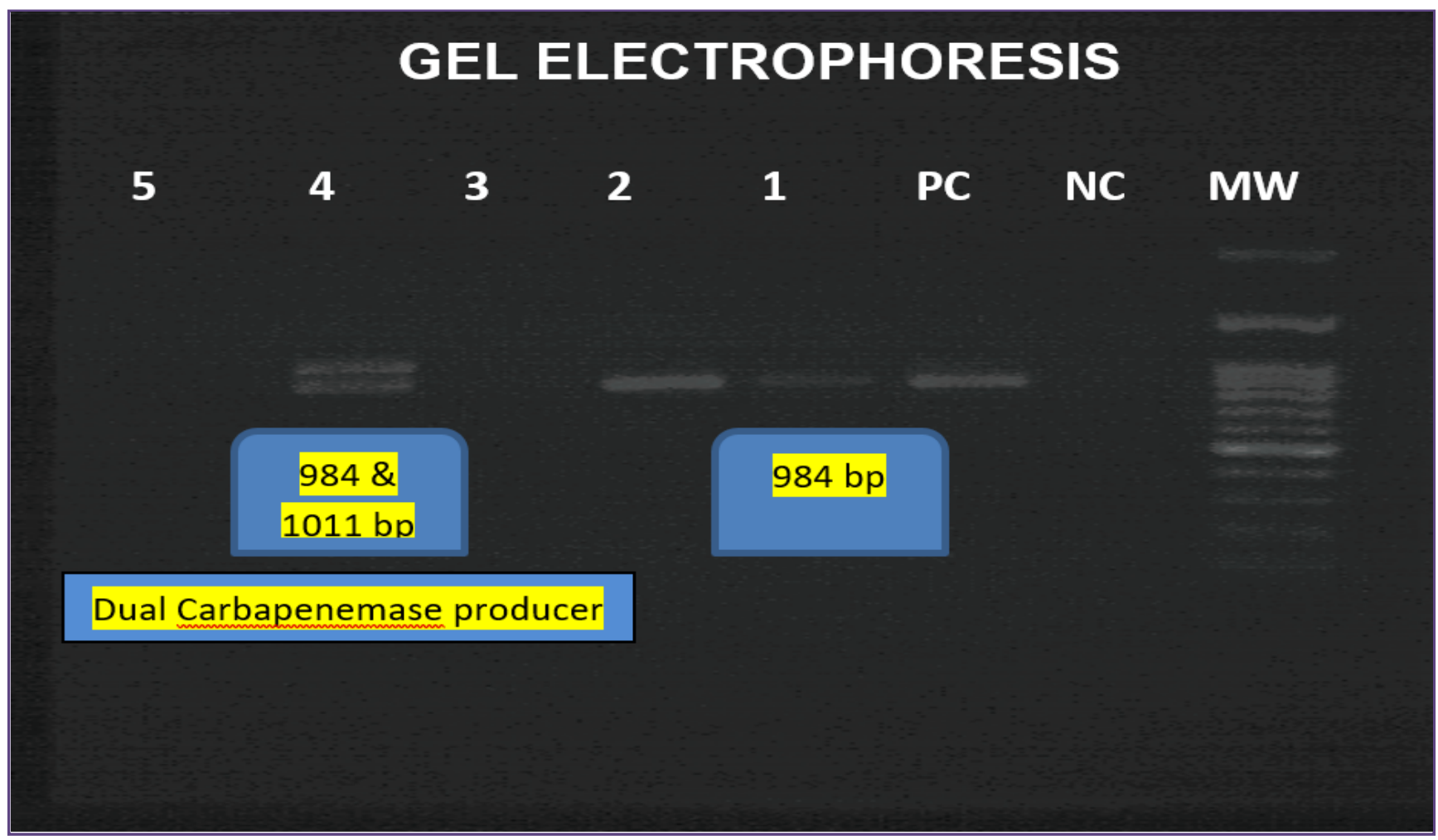

Fig. 6: Gel Electrophoresis II PC-Positive control NC- Negative control MW-molecular weight ladder. 
followed by 55 which were Klebsiella spp (16\%) 53 were Pseudomonas (15\%) and 30 were Acinetobacter baumanii $(8.6 \%)$ respectively.

Study by Mulla et al. and Patel et al. had similar finding which had E. coli and Klebsiellae as the most common isolates of Enterobacteriaceae in their clinical samples ${ }^{[5}$,

${ }^{6}$. The prevalence of carbapenem resistance was $31.14 \%$ in this study. Similarly, overall meropenem resistance was reported by Mulla $\mathrm{S}$ et al ${ }^{[5]}$ and Mahajan $\mathrm{G}$ et al ${ }^{[7]}$.

Majority of sample was of urine 63\% (220/350) followed by pus $21 \%$. Similar findings were reported in a comparable study by Mohamudha R.P et al ${ }^{[8]}$. In our study, carbapenem resistant organism was isolated mainly from urine $44.95 \%$ $(\mathrm{n}=49)$ followed by pus $22.93 \%(\mathrm{n}=25)$ and respiratory samples $18.34 \%(n=20)$. In a study by Nagaraj $S$ et al [9] they observed that carbapenem-resistant organisms were isolated mainly from urine samples up to $42 \%$ ( $n=$ 21 ), followed by wound discharge $18 \%$ and respiratory secretions $16 \%$.

Urine was the most frequent sample received during our study and in most of the studies analyzed. This may be attributed to urinary tract infection (UTI), being the most common hospital-acquired infection, which accounts for almost $40 \%$ of all nosocomial infections.

The meropenem-resistant isolates (109 out of a total of 350 isolates) were subjected to multiplex PCR for detection of resistance genes $b l a_{\mathrm{NDM}-1}, b l a_{\mathrm{VIM}}, b l a_{\mathrm{IMP}}, b l a_{\mathrm{OXA}}$ and $b l a_{\mathrm{KPC}}$. In addition 50 samples which were sensitive to Carbapenems by the disc diffusion method were randomly selected and subjected to multiplex PCR.

All 109 isolates which were resistant to Carbapenems by the disc diffusion method came positive for presence of Carbapenemase genes by PCR. NDM was detected in 54 samples (49.5\%), KPC in 38 samples (34.8\%), OXA-48 type in 18 samples $(16.5 \%)$ and VIM came positive for 03 samples $(2.7 \%)$ respectively. IMP gene was not detected in any of the isolates. In addition 04 isolates came positive for both $b l a_{\mathrm{NDM}-1}$ and $b l a_{\mathrm{KPC} .}$ The 50 samples which were sensitive to carbapenems by the disc diffusion method were negative for the presence of Carbapenemase genes. In other studies prevalence of blaNDM-1 producers among carbapenem-resistant Enterobacteriaceae ranged between $31.2 \%$ and $91.6 \%{ }^{[10]}$. Shanmugam et al in India reported a prevalence of $67.4 \%$ blaKPC gene in resistant Enterobacteriaceae ${ }^{[1]}$. In another study by S. Mohanty the prevalence of NDM-1 was $65.6 \%$, OXA-48 $24.7 \%$, OXA- 181 in $23.6 \%$, VIM was $6.4 \%$ and KPC was $2.1 \%$ respectively ${ }^{[12]}$.

\section{Conclusion}

The past few years have seen an unprecedented increase in the number of carbapenemase producing bacteria being identified from clinical isolates worldwide. Majority are multidrug resistant, causing prolonged and life-threatening infections. The carbapenemase producing genes are generally borne on transposable genetic elements or plasmids, capable of horizontal spread.

Preventing emergence and spread of these pathogens through strict infection control practices, judicious use of antibiotics, and early and timely detection especially in a hospital setting are of utmost importance. A sound infection control protocol, rational use of antibiotics and a good antimicrobial policy would not only reduce prevalence of these super bugs but would also contribute in extending the longevity of the carbapenems, the last resort antibiotics in many life-threatening infections.

\section{Acknowledgements}

None.

\section{Funding}

None.

\section{Competing Interests}

None declared.

\section{Reference}

1. Alba J, Ishii Y, Thomson K, Moland ES, and Yamaguchi $\mathrm{K}$. Kinetics study of KPC-3, a plasmid-encoded class A carbapenem-hydrolyzing $\beta$-lactamase. Antimicrob Agents Chemother. 2005; 49:4760-2.

2. Bratu S, Landman D, Haag R, Recco R, Eramo A, Alam $\mathrm{M}$ et al. Rapid spread of carbapenem-resistant Klebsiella pneumoniae in New York City: a new threat to our antibiotic armamentarium. Arch Intern Med. 2005; 165:1430-5.

3. Woodford N, Tierno Jr. PM, Young K, Tysall L, Palepou MF,and Ward E et al. Outbreak of Klebsiella pneumoniae Producing a New Carbapenem-Hydrolyzing Class A $\beta$-Lactamase, KPC-3, in a New York Medical Center. Antimicrob Agents Chemother. 2004; 48(12):4793-9.

4. Yigit H, Queenan AM, Anderson GJ, Domenech-Sanchez A, Biddle JW, Steward CD et al. Novel carbapenem-hydrolyzing beta-lactamase, KPC-1, from a carbapenem-resistant strain of Klebsiella pneumoniae. Antimicrob Agents Chemother. 2001; 45:1151-61.

5. Charan J, Mulla S, Panvala T. Antibiotic sensitivity of Enterobacteriaceae at a tertiary care center in India. Chronicles of Young Scientists. 2011;2(4):214.

6. J Patel, J Bhatt, V Javiya, K Patel. Anti-Microbial Susceptibility Patterns of Enterobacteriaceae Isolated From A Tertiary Care Unit In Gujarat. The Internet Journal of Microbiology [Internet]. 2009;6(1). 
7. Mahajan G, Sheemar S, Chopra S, Kaur J, Chowdhary D, Makhija S K. Carbapenem resistance and phenotypic detection of carbapenemases in clinical isolates of Acinetobacter baumannii. Indian J Med Sci. 2011;65:18-25.

8. Mohamudha Parveen R, Harish B, Parija S. Emerging Carbapenem Resistance Among Nosocomial Isolates of Klebsiella Pneumoniae in South India. Inter Journal Pharma and Bio Sci. 2010;6(2).

9. Macaden R, Chandran S, Nagaraj S, Shamanna P. Carbapenem resistance among Escherichia coli and Klebsiella pneumoniae in a tertiary care hospital in south India. Indian Journal of Medical Microbiology. 2012;30(1):93.

10. Kumarasamy KK, Toleman MA, Walsh TR, Bagaria J, Butt F, Balakrishnan, et al. Emergence of a new antibiotic resistance mechanism in India, Pakistan and the UK: a molecular, biological, and epidemiological study. Lancet Infect Dis 2010;10:597e602.

11. Shanmugam P, Meenakshisundaram J, Jayaraman P. BlaKPC gene detection in clinical isolates of carbapenems resistant Enterobacteriaceae in a tertiary care hospital. J Clin Diagn Res 2013;7:2736e8.

12. Mohanty S, Gaind R. In vitro susceptibility of carbapenemresistant Enterobacteriaceae to colistin: A hope at present. Indian J Med Microbiol 2016;34:558-60.

13. Clinical and Laboratory Standards Institute. 2016. Performance standards for antimicrobial susceptibility testing; 26th informational supplement. CLSI M100-S26. Clinical and Laboratory Standards Institute, Wayne, PA.

14. Miriagou V, Tzouvelekis L, Rossiter S, Tzelepi E, Angulo F, Whichard J. Imipenem Resistance in a Salmonella Clinical Strain Due to Plasmid-Mediated Class A Carbapenemase KPC-2. Antimicrobial Agents and Chemotherapy. 2003;47(4):1297-1300.

*Corresponding author:

Maj Parijat Das, Dept of Pathology Military Hospital, Defence Colony, Ekkatathangal, Chennai 600032, Tamil Nadu, India.

Phone: +91 7030731188

Email: parijat5628@gmail.com

Date of Submission $\quad: \quad 26 / 03 / 2021$

Date of Final Revision : $28 / 04 / 2021$

Financial or other Competing Interests: None.

Date of Acceptance $\quad$ : $\quad 20 / 05 / 2021$

Date of Publication $\quad$ : $30 / 05 / 2021$ 